\title{
Nanopartículas para el control del biodeterioro en monumentos históricos
}

\author{
M.A. Martínez Gómez*, M.C. González Chávez*, \\ J.C. Mendoza Hernández** ${ }^{*}$ R. Carrillo González*
}

\begin{abstract}
RESUMEN: La exposición directa de construcciones e inmuebles históricos al ambiente tiene efectos negativos en las propiedades de los materiales; debido al intemperismo físico y químico, pero los agentes biológicos catalizan su meteorización. Así, organismos y microflora en asociación desencadenan diversos procesos bioquímicos y físicos que inducen al biodeterioro del patrimonio cultural. El crecimiento de los microrganismos es difícil controlar sin causar daños físicos a los materiales colonizados. Actualmente, se buscan técnicas novedosas y eficaces para el control del biodeterioro que no dañen los monumentos, ni impliquen riesgos al ambiente. Una alternativa que ofrece amplias ventajas es el uso de nanopartículas de plata producidas a partir de extractos vegetales. Este documento discute ampliamente esta opción y evidencia las necesidades de desarrollo tecnológico al respecto para solucionar este problema.

PALABRAS CLAVE: síntesis verde, nanopartículas, microorganismos, monumentos.
\end{abstract}

ABSTRACT: The direct exposure of buildings and cultural heritage to the atmosphere has negative effects on the material properties due to physical and chemical weathering, but the biological agents catalyze disruption. Thus, association of organisms and microflora induces the biochemical and physical processes that develop biodeterioration of cultural heritage. Microbes become difficult to control without causing physical damage to the colonized materials. Currently, there are innovative and effective techniques in biological control which are environmental friendly. An alternative that offers many advantages is the application of silver nanoparticles produced from plant extracts. This paper widely discusses this option and shown the need of technological break through to face this problem.

KEY wORDS: green synthesis, nanoparticles, microrganisms, monuments.

\section{BIODETERIORO}

La conservación del patrimonio cultural de bienes inmuebles es transcendental debido a que representa la identidad del pasado, una expresión estética y utilitaria de las diferentes culturas humanas. Diversos autores mencionan que entre los principales procesos que participan en el deterioro del patrimonio cultural edificado están los: físicos, mecánicos de disgregación o fractura; químicos o de descomposición (Peraza-Zurita, 2004), biológicos (biodeterioro), así como los ocasionados por actividad humana directa (como vandalismo o saqueo) e, indirectamente, como la contaminación ambiental (Videla et al., 2003). El biodeterioro altera las características de los materiales porque incluye procesos bioquímicos que modifican su estética. La mi-

\footnotetext{
* Colegio de Postgraduados Campus Montecillo, km 36.5 Carretera México-Texcoco, 56230; Texcoco, México.

** Benemérita Universidad Autónoma de Puebla, 4 sur 104 72000, Puebla, México.

Correo-e de R. Carrillo González: crogelio@colpos.mx
} 
croflora y organismos en asociación presentes en fachadas, cimientos y monumentos al aire libre constituyen una variable difícil de controlar. Lo anterior, debido a sus formas de reproducción, colonización y metabolismo.

En los materiales pétreos, el proceso de biodeterioro se puede observar en el desgaste del material, la presencia de variaciones cromáticas antiestéticas, crecimiento de microrganismos (Páramo y Narváez, 2011) e inclusive en el crecimiento de plantas. Diversas variables favorecen la actividad microbiana desarrollada en sustratos de roca. Entre éstas se incluyen: propiedades físicoquímicas del sustrato, adherencia de los microrganismos (Arroyo et al., 2011), naturaleza de los nutrientes del soporte (Nieves, 2003), formación de biopelículas y asociaciones mutualistas (Scheerer et al., 2009). Sin embargo, entre los factores más importantes están la temperatura, la humedad y la exposición a la luz solar (Peraza-Zurita, 2004).

El término biorreceptividad permite definir el conjunto de propiedades que hacen susceptible un material a la colonización biológica (Miller et al., 2006). De esta forma, es importante determinar la capacidad de colonización de los organismos que originan biodeterioro sobre soportes mineralógicos específicos y bajo ciertas condiciones climáticas y ambientales (Nuhoglu et al., 2006).Diversos trabajos muestran que los productos metabólicos y células muertas de microrganismos fotosintéticos promueven el desarrollo de heterótrofos y plantas inferiores sobre ciertos soportes (figura 1). Lo anterior incrementa el detrimento físico y químico de la roca por acción sinérgica (Peraza-Zurita, 2004; Miller et al., 2006), por lo cual, los organismos invasores de los sustratos varían dependiendo del ambiente. Al respecto, Marcos-Laso (2001) expuso que en la ciudad de Salamanca, España, la diversidad liquénica que crece sobre

FIGURA 1. Esquema de la sucesión de especies que causan el biodeterioro (Fuente propia)

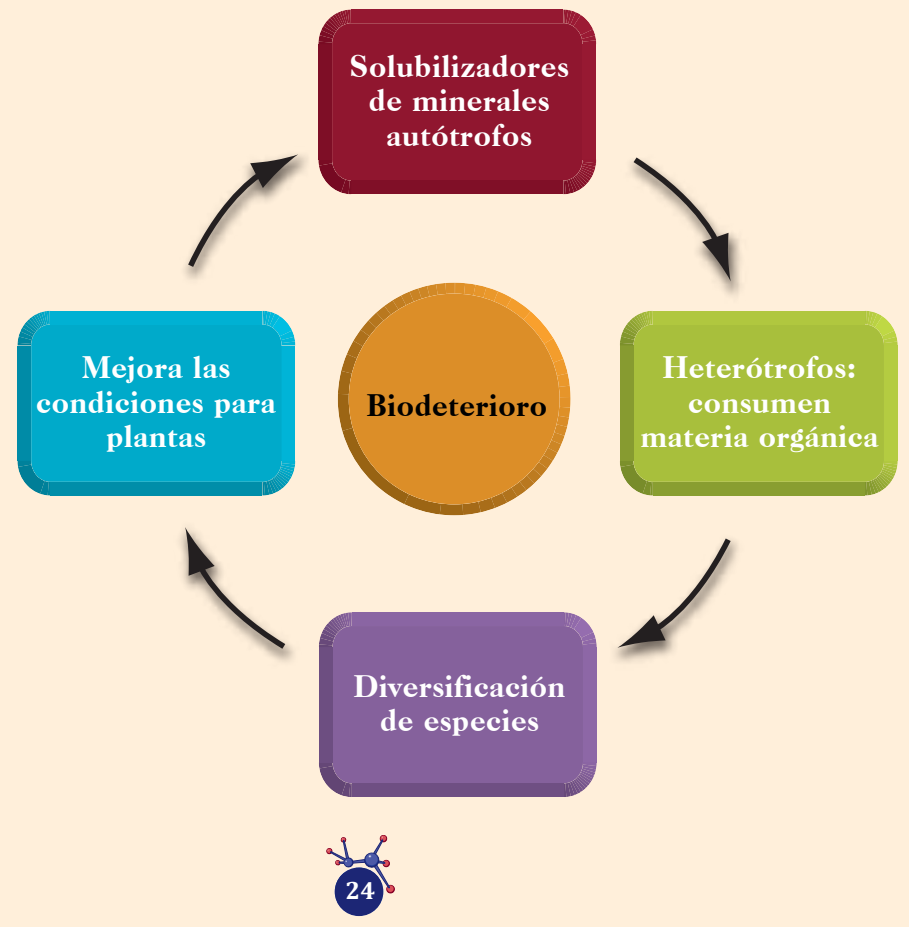


el puente romano, y cómo difiere de las especies colonizadoras de las paredes de las catedrales románica y gótica de la ciudad, a pesar de ser sus materiales de construcción similares.

Entre las consecuencias de la actividad biológica sobre sustratos rocosos están la solubilización de silicatos, carbonatos y sulfatos, alteración por cristalización de sales y diferencias entre las presiones de hidratación (Caneva et al., 2000). Antes de intervenir objetos o monumentos históricos debe realizarse un análisis integral con el fin de determinar si los organismos presentes constituyen un riesgo potencial. Además, conocer las estrategias reproductivas de las especies involucradas en el biodeterioro permite establecer las causas de su crecimiento, definir estrategias oportunas para su control y prever las consecuencias de su erradicación. En este sentido, es conveniente resaltar que cuando los materiales de construcción forman parte de obras de interés patrimonial, cualquier alteración tiene repercusión en aspectos históricos y culturales e, inclusive, en términos económicos y turísticos.

\section{MICRORGANISMOS DEL BIODETERIORO}

La identificación de microrganismos que colonizan los materiales de construcción, proporciona información esencial para cualquier proyecto de investigación cuyo objetivo sea el manejo del biodeterioro (Fonseca et al., 2010). En el proceso de biodeterioro intervienen bacterias quimiolitotróficas, autotróficas y heterotróficas, hongos, algas, líquenes, musgos y plantas superiores. Entre las bacterias que atacan los monumentos y edificios de piedra se pueden mencionar tio bacterias, silico bacterias y bacterias nitrificantes (Videla et al., 2003). Estas bacterias pueden, entre otras cosas, disolver minerales, convertir nitratos en nitritos y sulfatos en sulfuros; de este modo son capaces de producir ácido nítrico, nitroso y sales de amonio que provocan alteraciones en los materiales de construcción.

Flores et al. (1997) reportaron en monumentos de la ciudad de Alcalá de Henares, España, crecimiento de los géneros bacterianos Bacillus, Micrococcus y Thiobacillus; así como de microalgas del género Apatococcus. Su estudio propuso el empleo de diversas técnicas analíticas para demostrar la actividad microbiana sobre superficies rocosas. Además, confirmó que las costras pétreas de monumentos y esculturas de roca son sustratos microbianos que ofrecen posibilidades nutricionales y de interacción entre distintos tipos de poblaciones. Ejemplo de lo anterior, se observó en diversos sitios arqueológicos de India, donde se aislaron más de diecinueve géneros de hongos (Pandey et al., 2011), entre los que se encontraron: Beauveria sp., Bipolaris sp., Curvularia sp., Cochliobollus sp., Chaetomium sp., Crysosporium sp., Conidiobolus sp., Fusarium sp. y Penicillium sp.

Estudios de los sitios arqueológicos de Uxmal y Tulum en el área maya determinaron que las rocas calizas de los monumentos difieren básicamente en su color y porosidad. Además, la colonización de la superficie rocosa por microrganismos se presenta con base en la ubicación de las paredes (internas o externas) y tiempo de exposición a luz (natural y artificial). Videla et al., (1993) mostraron que estas características determinan el grado de biorreceptividad de las rocas al biodeterioro. En este trabajo se identificaron bacterias de los géneros Pseudomonas sp. y Bacillus sp.; así como hongos Aspergillus sp. y Monilia sp. La pared interna del Templo del Viento en Tulum presentó mayor presencia de cianobacterias del género Gloeocapsa sp., Pleuro- 
capsa sp., Synechocystis sp. y Synechococcus sp.; mientras que en la pared externa de la Casa del Cenote de Tulum se observó Gloeocapsa sp. y Gloeothece sp.

En una publicación del Instituto de Investigaciones Históricas, Arqueológicas y Antropológicas de Guatemala (Aquino-Lara et al., 2009), se describieron los principales organismos que crecen sobre los componentes arquitectónicos del sitio arqueológico de Nakum. La identificación de musgos incluyó: Hyophila involuta, Barbula indica y Trichostomun sp. En relación con líquenes hubo presencia de dos géneros: Lepraria sp. y Graphis sp. Por otra parte, en el Castillo de Chapultepec, (Ciudad de México), construido durante elsiglo XVIII, se investigaron los agentes biológicos quecolonizaban sus paredes. Se comprobó la coexistencia de los hongos filamentosos: Cladosporium sp., Mucor sp., Alternaria sp., entre otros. Además de los géneros bacterianos Bacillus sp., Pantoea sp. y Kokuria sp. (Páramo y Narváez, 2011). Muchos de estos géneros microbianos se reportan frecuentemente como participantes en procesos de biodeterioro de monumentos.

Aunque el problema de la conservación integral de los inmuebles y monumentos es complejo, numerosas investigaciones extranjeras proponen métodos de desinfección química. Sin embargo, la interacción de ciertas sustancias, algunas de ellas tóxicas, como el óxido de etileno y el bromuro de metilo (EPA, 2012), con los materiales, puede generar reacciones químicas y productos que inducen un mayor deterioro en los materiales (Ascasoa et al., 2002; Fonseca et al., 2010). Asimismo, el costo y eficacia de los tratamientos no siempre es el esperado. Aunado a esta problemática, en México son escasos los estudios formales sobre el control del biodeterioro. Sin embargo, se buscan técnicas novedosas y eficaces en el control de microrganismos, que no alteren significativamente el material original; que inhiban el crecimiento de los organismos y sean amigables con el ambiente; una alternativa que ofrece amplias ventajas es el uso de nanopartículas.

\section{NANOPARTÍCULAS}

La nanotecnología es una especialidad de amplio desarrollo en los últimos años debido a sus diversas aplicaciones y alcances: desde la medicina hasta la industria y recientemente la nanociencia en la conservación del patrimonio histórico. Gracias a las investigaciones sobre la alteración del color sufrida por las partículas metálicas de diversas reliquias, se planteó la posibilidad de la aplicación de nanopartículas dentro del campo de la conservación de los materiales. De esta manera, se eligió la aplicación de nanopartículas en emulsión para solucionar o prevenir los problemas del biodeterioro de superficies de monumentos y construcciones históricas (Gómez-Villalba, 2011; Baglioni y Giorgi, 2006).

Un nanomaterial se define como aquel que posee ciertas características estructurales de las cuales al menos una de sus dimensiones está en el intervalo de 1-100 nanómetros $\left(1 \mathrm{~nm}=1 \times 10^{-9} \mathrm{~m}\right)$. El cambio en tamaño proporciona a las nanoestructuras nuevas propiedades y comportamientos. Como, por ejemplo, una partícula de tamaño nanométrico provee un área específica superficial mayor para la colisión molecular y, por tanto, incrementa su velocidad de reacción (Gómez-Villalba, 2011). Esto sucede porque las partículas nanométricas manifiestan ciertas características químicas y físicas asociadas a su tamaño (Poole y Owens, 2007; Philip, 2009; Baglioni et al., 2009).

Existe una creciente necesidad de desarrollar técnicas de síntesis que disminuyan el uso de químicos para su producción (Jae y Beom, 2009). No obstante, el enfoque 
químico para la producción de nanopartículas es el más popular, aunque no puede evitar el uso de químicos tóxicos en sus protocolos de síntesis. La química verde es una alternativa a la química convencional porque utiliza biomasa vegetal, microrganismos, enzimas y extractos de plantas (Yonghong et al., 2009) para la producción de nanomateriales. Esta forma de síntesis resulta no tóxica y amigable con el ambiente. Las ventajas de estos procedimientosse reflejan en el costo, menor toxicidad para el ambiente y desaparición de procesos de polimerización durante la síntesis, entre otras (Maensiri et al., 2008).

Numerosos trabajos proponen el uso de extractos de plantas como precursores naturales en la síntesis de nanopartículas. Jaey Beom (2009); Philip (2009); Sathishkumar (2009); Antariksh et al., (2010); Singhalet al. (2011); y, ChidambaramyAbdul (2012) demostraron que el uso de plantas en el proceso de síntesis ofrece mayores ventajas con respecto a otros procesos biológicos. Roy et al., (2013) publicaron un estudio sobre la síntesis de nanopartículas de plata por vía microbiana. Este trabajo reveló que la enzima extracelular reductasa, producida por el hongo Aspergillus foetidus MTCC8876, conduce a la formación de nanopartículas. No obstante, su funcionalidad, el material microbiano para la formación de nanopartículas en muchas ocasiones implica el desarrollo de líneas celulares de alto costo (Jae y Beom, 2009). En el caso de los extractos de plantas, éstos funcionan como agentes inductores de la síntesis, confiriéndoles estabilidad y durabilidad durante periodos más prolongados (Maensiri et al., 2008).

Actualmente, se reportan más de cien especies de plantas cuyas infusiones y extractos acuosos confieren ventajas al proceso de síntesis de nanopartículas puras de diversos elementos. Al respecto, se publicó un estudio comparativo de cuatro extractos de hojas de plantas de: pino (Pinussylvestris), gingko (Ginkgo biloba L.), magnolia (Magnolia grandiflora L.) y persimmon (Diospyros kaki) útiles en la síntesis extracelular de nanopartículas metálicas de plata (Jae y Beom, 2009). El extracto de hojas de magnolia mostró mayor capacidad como agente reductor en términos de síntesis y conversión a $\mathrm{Ag}^{0}$. Este trabajo concluye que el tamaño promedio de las nanopartículas (30 $\mathrm{nm}$ ) depende de las condiciones de temperatura y velocidad de reacción, el tipo y concentración del extracto así como la concentración de $\mathrm{AgNO}_{3}$.

\section{NANOPARTíCULAS EN EL CONTROL DEL BIODETERIORO}

Las nanopartículas presentan características específicas que les permiten interactuar con los microrganismos que colonizan la superficie de diferentes materiales como: aplanados, piedra, madera, metales, etc. Tales interacciones desencadenan mecanismos de inhibición microbiana (Liet al., 2008), de suma importancia en el control biológico de organismos. Recientemente, algunos nanomateriales naturales y artificiales manifestaron diversas propiedades antimicrobianas y biocidas, como quitosano, óxido de titanio fotocatalítico, fullerol, nanotubos de carbono, nanopartículas acuosas de fuereños y de plata (Liu et al., 2012). A diferencia de los desinfectantes químicos convencionales, estos nanomateriales antimicrobianos no son oxidantes fuertes y son relativamente inertes en agua. Por esta razón no se espera que ocasionen efectos perjudiciales en el ambiente ni a la salud humana.

De acuerdo con Li et al. (2008); las nanopartículas de péptidos, quitosano, carboxifulereno, nanotubos de carbono, óxido de zinc y de plata alteran la envoltura celular bacteriana. Además, la presencia de nanopartículas de plata altera el proceso de transporte electrónico en el interior de la célula (figura 2). Lo anterior desencade- 
FIGURA 2. Las nanopartículas de plata interactúan con los grupos tiol de proteínas en la célula, lo cual produce oxidación de proteínas e inactiva el sistema respiratorio celular. Asimismo, estas nanopartículas afectan el funcionamiento y permeabilidad de la membrana en diversos microrganismos como mecanismo de acción biocida. En consecuencia, hay alteraciones en transporte de $\mathrm{K}^{+}$y $\mathrm{Na}^{+}$; así como en la unión del Fosfato de Adenosina (ADP) con la molécula de fósforo $\mathrm{P}_{\mathrm{i}}$ para formar Trifosfato de Adenosina (ATP), en el interior de la célula (mitocondria). De este modo, se reduce el contenido de esta molécula de importancia energética (Fuente propia).

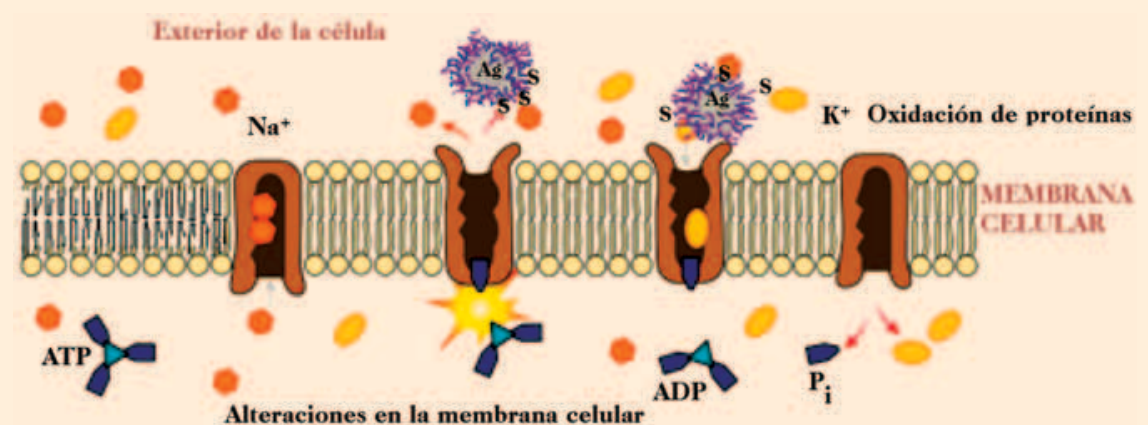

na alteraciones enzimáticas, ruptura de la membrana o pared celular de los microrganismos y, finalmente, daños irreversibles en el DNA (Pal et al., 2007; Liet al., 2008; Singhal et al., 2011). Adicionalmente, recientes investigaciones demuestran que el uso de nanopartículas de fullereno acuoso y las nanopartículas de plata interrumpen la transducción de energía transmembranal en la células microbianas (Zhang y Wang, 2007). Esto implica que estas partículas, no sólo matan a los organismos ya establecidos sobre el material a tratar, sino también otros que quieran establecerse, mientras se mantengan activas.

Las propiedades antimicrobianas y biocidas de compuestos e iones de plata (JuNamy Lead 2008; Chidambaram y Abdul, 2012; Gopinatha et al., 2012; MarambioJones y Hoek, 2010; Jain et al., 2009) se conocen desde la antigüedad bajo una amplia gama de aplicaciones, tales como: agentes de tinción para matrices biológicas y de microscopía electrónica, y, recientemente, como agente de inhibición de biopelículas microbianas (Kalishwaralal et al., 2010), en el tratamiento del agua(Zhiya-Sheng y Yang-Liu, 2011; De Santa María et al., 2010). También, existen trabajos que revelan la capacidad citotóxica de las nanopartículas de plata (Dipankar y Murugan, 2012; Ravindranet al., 2013; Gengan et al., 2013), con prometedoras aplicaciones biomédicas. Sin embargo, este mecanismo no está completamente descrito (Ju-Nam y Lead, 2008).

Las propiedades fisicoquímicas de las nanopartículas plata le confieren una importante actividad antimicrobiana. De especial interés es el tamaño (Martínez-Castañón et al., 2008). En general, las partículas menores de $10 \mathrm{~nm}$ resultan tóxicas para bacterias como Escherichia coli y Pseudomonas aeruginosa. Los iones de plata interactúan con los grupos tiol de las proteínas, dando como resultado una alteración de procesos celulares e inactivación de las enzimas respiratorias. Los iones de plata son también fotoactivos en presencia de radiación ultravioleta (UV-A y UV-C), lo cual genera la inactivación de bacterias y virus (Liet al., 2008). Otros estudios sobre degradación de la membrana celular en presencia de moléculas de plata determinaron que 
éstas estimulan el aumento en la permeabilidad de la membrana celular (Zhang y Wang, 2007; Pal et al., 2007).

Gran parte del deterioro de los materiales rocosos se relaciona con propiedades intrínsecas, como textura y composición, porosidad y grado de alteración de los minerales presentes. Además, como se señaló al inicio, existe una serie de factores extrínsecos, naturales o antropogénicos, cuya relación con el ambiente contribuye a la degradación química y física de los materiales. Existen productos cuyo principio activo se basa en nanopartículas y nanoemulsiones de hidróxidos de calcio, magnesio, estroncio, ferrita, óxido de silicio, magnesio, zinc y plata, por mencionar algunos ejemplos, cuya aplicación se encamina a la conservación de piedra, lienzos, madera, papel y morteros entre otros (Baglioni y Giorgi, 2006; Gómez-Villalba, 2011). Ciertos nanoproductos, son capaces de producir efecto biocida, resultando útil para el control del biodeterioro de materiales pétreos.

En 2012, se publicaron los resultados del análisis de contaminación microbiana del aire y superficies en seis museos de Polonia. Durante este estudio, se evaluó el grado de sensibilidad a nanopartículas de plata por parte de microrganismos con mayor presencia en distintos objetos, utilizando 32 cepas bacterianas y fúngicas (Gutarowska et al., 2012). El tamaño de las nanopartículas de plata producidas bajo síntesis química fue de 10 a $100 \mathrm{~nm}$, definiéndose su concentración eficaz para la eliminación de microrganismos presentes en la superficie de los objetos en 45 ppm. Los resultados del experimento permitieron la eliminación del 94\% de los microrganismos presentes, excepto en el caso de las cepas de Bacillus subtilis y Staphylococcus xylosus.

En otras experiencias, se probó la capacidad biocida de las nanopartículas de plata sintetizadas con Ocimum sanctu (Singhal et al., 2011), así como bacterias E. coli (Gram negativas) y $S$. aureus (Gram positivas). En este trabajo, se observó que el incremento en la concentración de nanopartículas de biosíntesis (10-25 nm) disminuyó el crecimiento de ambas especies microbianas. La adición del extracto en la solución de nitrato de plata favoreció la reducción de iones de plata en nanopartículas. Lo anterior probablemente se relaciona con la presencia de proteínas que actúan como agente de término (Singhalet al., 2011).Otro trabajo que confirma la funcionalidad antibacteriana de las nanopartículas de biosíntesis, se realizó a partir de extractos de cebolla (Allium cepa). La actividad antibacteriana se estudió frente a E. coli y S. typhimurium (Antariksh et al., 2010). Aquí se concluyó que una concentración de $50 \mu \mathrm{g} \mathrm{mL}{ }^{-1}$ de nanopartículas de plata en solución resultó eficaz contra el desarrollo de estas bacterias.

Por otro lado, una investigación, realizada con nanopartículas de plata obtenidas a partir de síntesis biológica con extractos de corteza de canela (Cinnamon zeylanicum), mostró inhibición del crecimiento de E. coli BL-21. Sathishkumar et al., (2009) y Philip (2009) concluyeron que el pH influye en el control del tamaño de las partículas. En este sentido, se sabe que el tamaño (cuadro 1) tiene efectos importantes en el efecto antimicrobiano (Ashkarran et al., 2012). En coincidencia con lo demostrado por Pal et al. (2007), quienes, con imágenes de microscopía electrónica de transmisión, revelaron que la forma de las nanopartículas de plata influye en la actividad biocida contra E. coli. Tal es el caso de las nanopartículas en placas triangulares truncas al mostrar una fuerte acción biocida, en comparación con las nanopartículas con formas esférica y de barra.

Aunque existen desventajas y posibles efectos referentes a la aplicación de nanopartículas dadas las características fitotóxicas de algunas moléculas metálicas como la plata, numerosos estudios apoyan su aplicación con fines ambientales. Tal es el caso 
CUADRO 1. Formas de nanopartículas de plata reportadas por diversos autores

\begin{tabular}{|l|l|l|l|}
\hline & \multicolumn{1}{|c|}{$\begin{array}{c}\text { Forma de NP } \\
\text { de plata }\end{array}$} & $\begin{array}{c}\text { Actividad } \\
\text { antimicrobiana }\end{array}$ & \multicolumn{1}{c|}{ Referencia } \\
\hline & Esférica (nanosphere) & S. aureus y E. coli & Pal S. et al., 2007 \\
\hline & Barra (nanorod) & E. coli & $\begin{array}{l}\text { Jiu J. et al., 2011; } \\
\text { Van Dong et al., 2012 }\end{array}$ \\
\hline & $\begin{array}{l}\text { Placa triangular trunca } \\
\text { (nanotriangle) }\end{array}$ & $\begin{array}{l}\text { E. coli, S. aureus y } \\
\text { P. aeruginosa }\end{array}$ & $\begin{array}{l}\text { Kelly J. et al., 2012; } \\
\text { Gao M. et al., 2013 }\end{array}$ \\
\hline $\begin{array}{l}\text { Poliédrica (polyhedral } \\
\text { silver nanoparticles) }\end{array}$ & $\begin{array}{l}\text { Reducción de oxígeno } \\
\text { en medio alcalino }\end{array}$ & Kuai L. et al., 2011 \\
\hline
\end{tabular}

Fuente propia.

del trabajo realizado por Lee et al., (2012); en el cual comparó la toxicidad y biodisponibilidad de nanopartículas de plata en cultivos de Phaseolus radiatus y Sorghum bicolor sobre agar y suelo. En agarencontraron una correlación entre el crecimiento de las plántulas de estas especies y la concentración de las nanopartículas de plata que se aplicó en cada ensayo. Por el contrario, en el suelo la tasa de crecimiento de P. radiatus no mostró afectaciones por la presencia de diferentes concentraciones de nanopartículas con respecto a las pruebas con agar.

En este trabajo, concluyeron que la biodisponibilidad de las nanopartículas se redujo en el suelo debido a que las nanopartículas exponen propiedades fisicoquímicas específicas en sistemas minerales, gracias a la capacidad tampón del suelo. Los electrolitos presentes en el suelo interactúan con las nanopartículas de plata, lo cual incrementa el estado de agregación de las nanopartículas y, en consecuencia, evita su paso hacia la raíz de las plantas (Leeet al., 2012). Además, la arcilla es capaz de retener Ag debido al fenómeno de intercambio catiónico, así como a formar enlaces S-A-S con azufre inorgánico y orgánico (Wijnhoven et al., 2009).

Es importante señalar los escasos estudios realizados en México sobre la aplicación de nanopartículas para el control de microrganismos que originan biodeterioro. No obstante, resulta esencial, no sólo evaluar el efecto inhibitorio de las nanopartículas sobre los microrganismos que crecen sobre los materiales, sino también considerar la estabilidad y comportamiento de las nanopartículas sobre diferentes sustratos. Lo anterior permitirá analizar la generación de productos secundarios ante diversos factores, así como responder las interrogantes surgidas sobre la funcionalidad y eficacia de esta tecnología a largo plazo.

\section{ConCLUSIÓN}

La conservación del patrimonio cultural es importante desde el punto de vista de identidad histórica y cultural, estética de los sitios arqueológicos, y práctica por el turismo; a pesar de que se han estudiado las causas, particularmente las biológicas, no se han generado procedimientos de control totalmente efectivos. En este contexto el 
uso de nanopartículas para el control del biodeterioro, parece tener ventajas particulares, al comparar con otras técnicas de control, sin embargo aún hay varias preguntas técnicas que resolver y respecto a su relación costo beneficio y riesgos que conlleva.

\section{REFERENCIA}

Antariksh, S.; Tripathi, R. y Singh, R. (2010) "Biological synthesis of silver nanoparticles by using onion (Allium cepa) extract and their antibacterial activity". Digest Journal of Nanomaterials and Biostructures, 5: 427-432.

Ashkarran, A.; Ghavami, M.; Aghaverdi, H.; Stroeve, P. y Mahmoudi, M. (2012) "Bacterial effects and protein corona evaluations: Crucial ignored factors in the prediction of bio-efficacy of various forms of silver nanoparticles". Chemical Research in Toxicology, 25: 1231-1242.

Aquino-Lara, D.; Barrios-Villar, A.; Ríos-Gálvez, M. y Espigares-Luarca, C. (2009) Crecimiento y distribución de organismos vegetales menores en la arquitectura prehispánica y su relación con el deterioro del patrimonio cultural edificado. Guatemala: Universidad de San Carlos de Guatemala, Dirección General de Investigación, Programa Universitario de Investigación en Cultura, Escuela de Historia, IIHAA: 158.

Arroyo, I.; Sarró, I. y Montero, J. (2011) “Peculiaridades del estudio y control del biodeterioroen cuevas con arte rupestre”. En S.G.T. Ministerio de Cultura, ed. La Ciencia y el Arte III. Primera ed. Madrid: Instituto del Patrimonio Cultural de España.

Ascaso, C.; Wierzchos, J.; Souza-Egipsy, V.; Ríos, A. y Delgado-Rodrigues, J. (2002) "In situ evaluation of the biodeteriorating action of microrganisms and the effects of biocides on carbonate rock of the Jeronimos Monastery (Lisbon)". International Biodeterioration and Biodegradation, 49: 1-12.

Baglioni, P. y Giorgi, R. (2006) "Soft and hard nanomaterials for restoration and conservation of cultural heritage". The Royal Society of Chemistry, 2: 293-303.

Baglioni, P.; Giorgi, R. y Dei, L. (2009) "Soft condensed matter for the conservation of cultural heritage". Comptes Rendus Chimie, 12: 61-69.

Caneva, G.; Nugar, M. y Salvadori, O. (2000) La biología en la restauración. 2a. ed. Sevilla: Nerea.España.

Chidambaram, J. y Abdul, R. (2012) “Acaricidal efficacy of synthesized silver nanoparticles using aqueous leaf extract of Ocimum canum against Hyalomma anatolicum anatolicum and Hyalomma marginatum isaaci". Parasitology Research, 111: 369-1378.

De Santa Maria, L.; Oliveira, R.; I; Merçon, F.; Borges, M.; Barud, Ribeiro, S.; Messaddeq, Y. y Wang, S. (2010) "Preparation and bactericidal effect of composites based on crosslinked copolymers containing silver nanoparticles". Polímeros, 20: 227-30.

Dipankar, C. y Murugan, S. (2012) "The green synthesis, characterization and evaluation of the biological activities of silver nanoparticles synthesized from Iresine herbstii leaf aqueous extracts". Colloids and Surfaces B: Biointerfaces, 98: 112-19.

EPA (2012) Programas de seguridad con los pesticidas. U.S. Environmental Protection Agency. <http://www.epa.gov/oppfead1/safety/spanish/>.

Flores, M.; Lorenzo, J. y Gómez, G. (1997) "Algae and bacteria on historic monuments at Alcala de Henares, Spain". International Biodeterioration and Biodegradation, 40: 241-46. 
Fonseca, A.; Pina, F.; Macedo, M. y Leal, N. (2010) "Anatase as an alternative application for preventing biodeterioration of mortars: Evaluation and comparison with other biocides". International Biodeterioration and Biodegradation, 64: 388-96.

Gengan, M.; Ananda, K.; Phulukdareeb, A. y Chuturgoonb, A. (2013) "A549 lung cell line activity of biosynthesized silver nanoparticles using Albizia adianthifolia leaf". Colloids and Surfaces B: Biointerfaces, 105: 87-91.

Gómez-Villalba, L. (2011) “Nanopartículas para la conservación del patrimonio”. En: Gómez-Villalba, L.S. La conservación de los geomateriales utilizados en el patrimonio. Madrid: Instituto de Geociencias (CSIC-USM).España.

Gopinath, V.; Mubarak Ali, D.; Priyadarshini, S.; Priyadharsshini, N.; Thajuddin, N. y Velusamy, P. (2012) "Biosynthesis of silver nanoparticles from Tribulus terrestris and its antimicrobial activity: A novel biological approach". Colloids and Surfaces B: Biointerfaces, 96: 69-74.

Gutarowska, B.; Skora, J.; Zduniak, K. y Rembisz, D. (2012) “Analysis of the sensitivity of microrganisms contaminating museums and archives to silver nanoparticles". International Biodeterioration and Biodegradation, 68: 7-17.

Jae, J. y Beom, S. (2009) "Rapid biological synthesis of silver nanoparticles using plant leaf extracts". Bioprocess Biosystems Engineering, 39: 79-84.

Jain, J.; Arora, S.; Rajwade, J.; Omray, P.; Khandelwal, S. y Paknikar, M. (2009) "Silver nanoparticles in therapeutics: Development of an antimicrobial gel formulation for topical use". Molecular Pharmaceutics, 6: 1388-1401.

Jiu, J.; Tokuno, T.; Nogi, M. y Suganuma, K. (2011) "Preparation and application rod-shaped and spherical silver nanoparticles". International Conference on Electronic Packaging Technology and High Density Packaging. Article number 6066823: 221-223.

Ju-Nam, Y. y Lead, J. (2008) "Manufactured nanoparticles: An overview of their chemistry, interactions and potential environmental implications". Science of the Total Environment, 400: 396-414.

Kalishwaralal, K.; BarathManiKanth, S.; Pandian, S.; Deepak, V. y Gurunathan, S. (2010) "Silver nanoparticles impede the biofilm formation by Pseudomonas aeruginosa and Staphylococcus epidermidis". Colloids and Surfaces B: Biointerfaces, 79: 340-44.

Kuai, L.; Geng, B.; Wang, S.; Zhao, Y.; Luo, Y. y Jiang, H. (2011) "Silver and gold icosahedra: One-pot water-based synthesis and their superior performance in the electrocatalysis for oxygen reduction reactions in alkaline media". European Journal Chemistry- $A$., 17: 3482-3489.

Lee W. M., L.; Jin-Il, K. y Youn-Joo, A. (2012) "Effect of silver nanoparticles in crop plants Phaseolus radiatus and Sorghum: Media effect on phytotoxicity". Chemosphere, 86: 491-99.

Li, Q.; Mahendra, S.; Lyon, D.; Brunet, L.; Liga, M.; Li, D. y Alvarez, P. (2008) “Antimicrobial nanomaterials for water disinfection and microbial control: Potential applications and implications". Water Research, 42: 4591-4602.

Liu, C.; Xie, X. y Cui, Y. (2012) "Antimicrobial nanomaterials for water desinfection”. NanoAntimicrobial. Progress and Prospects; Springer XVI: 465-494.

Maensiri, S.; Laokula, P.; Klinkaewnaronga, J.; Phokhaa, S.; Promarakc, V. y Seraphind, S., (2008) "Indium oxide $\left(\operatorname{In}_{2} \mathrm{O}_{3}\right)$ nanoparticles using Aloe vera plant extract: Synthesis and optical properties". Journal of Optoelectronics and Advanced Materials, 10: 16165. 
Marambio-Jones, C. y Hoek, E. (2010) “A review of the antibacterial effects of silver nanomaterials and potential implications for human health and the environment". Journal of Nanoparticles Research, 12: 1531-1551.

Marcos-Laso, B. (2001) “Biodiversidad y colonización liquénica de algunos monumentos en la ciudad de Salamanca (España)". Botanica Complutensis, 25: 93-102.

Martínez-Castañón, G.; Niño-Martínez, N.; Martínez-Gutiérrez, F.; Martínez-Mendoza, J. y Ruiz, F. (2008) "Synthesis and antibacterial activity of silver nanoparticles with different sizes". Journal of Nanoparticles Research, 10: 1343-1348.

Miller, A.; Dionisio, A. y Macedo, M.F. (2006) "Primary bioreceptivity: A comparative study of different portuguese lithotypes". International Biodeterioration and Biodegradation, 57: 136-142.

Nieves, V. (2003) "El biodeterioro de materiales orgánicos". En: El biodeterioro de materiales orgánicos. Instituto del Patrimonio Histórico Español. Madrid, España: Arbor.

Nuhoglu, Y.; Oguz, E.; Uslu, H.; Ozbek, A.; Ipekoglu, B.; Ocak, I. y Hasenekoglu, I. (2006) “The accelerating effects of the microrganisms on biodeterioration of stone monuments under air pollution and continental-cold climatic conditions in Erzurum, Turkey". Science of the Total Environment, 364: 272- 283.

Oromieh, G. (2011) "Evaluating solubility, aggregation and sorption of nanosilver particles and silver ions in soils", tesis de maestría en ciencias ambientales. Department of Soil and Environment, Swedish University of Agricultural Sciences.

Pal, S.; Kyung-Tak, Y.y Song, J. (2007) “Does the antibacterial activity of silver nanoparticles depend on the shape of the nanoparticle? A study of the gram-negative bacterium Escherichia coli". Applied and Environmental Microbiology, 73: 1712-1720.

Pandey, A.; Shrivastav, A. y Bhatnagar, P. (2011) “Diversity of monument deterioration-causing fungi at Gwalior Fort, India”. Annals of Environmental Science, 5: 35-40.

Páramo, L. y Narváez, J. (2011) “Aislamiento e identificación de microrganismos en biopelículas provenientes del Castillo de Chapultepec, Ciudad de México". Nexo, Revista Científica, 24: 83-91.

Peraza-Zurita, Y. (2004) Biodeterioro por microalgas en fuentes de mármol. Granada: tesis doctoral, Universidad de Granada.

Philip, D. (2009) "Green synthesis of gold and silver nanoparticles using Hibiscus rosa sinensis". Physica E, 11: 10-16.

Poole, C. y Owens, F. (2007) “Introducción a la física del estado sólido". En: Poole, C. y Owens, F. Introducción a la nanotecnología. Barcelona: Reverté. 128 pp.

Ravindran, A.; Chandran, P. y Khan, S. (2013) "Biofunctionalized silver nanoparticles: Advances and prospects”. Colloids and Surfaces B: Biointerfaces, 105: 342-352.

Roy, S.; Mukherjee, T.; Chakraborty, S. y Kumar-Das, T. (2013) "Biosynthesis, characterisation $y$ antifungal activity of silver nanoparticles synthesized by the fungus Aspergillus foetidus MTCC8876". Digest Journal of Nanomaterials and Biostructures, 8: 197-205.

Singhal, G.; Bhavesh, R.; Kasariya, K.; Ranjan-Sharma, A. y Pal-Singh, R. (2011) "Biosynthesis of silver nanoparticles using Ocimum sanctum (Tulsi) leaf extract and screening its antimicrobial activity". Journal of Nanoparticles Research, 13: 2981-2988.

Sathishkumar, M.; Sneha, K.; Won, S.W. y Cho, S.W. (2009) “Cinnamon zeylanicum bark extract and powder mediated green synthesis of nano-crystalline silver particles and its bactericidal activity". Colloids and Surfaces B: Biointerfaces, 73: 332-38. 
Scheerer, S.; Ortega-Morales, O. y Gaylarde, G. (2009) “Microbial deterioration of stone monuments". Advances in Applied Microbiology, 66: 93-139.

Van Dong, D.; Hoang Ha, C.; Binh, T. y Kasbohm, J. (2012) “Chemical synthesis and antibacterial activity of novel-shaped silver nanoparticles". International Nano Letters, 2: 9.

Videla, H.; Guiamet, P. y Gómez de Saravia, S. (2003) "Biodeterioro de materiales estructurales de sitios arqueológicos de la civilización maya”. Revista del Museo de la Plata, 44: 1-11.

Wijnhoven, S.; Peijnenburg, W.; Herberts, C.; Hagens, W.; Oomen, A.; Heugens, E.; Roszek, B.; Bisschops, J.; Gosens, I.; Van de Meent, D.; Dekkers, S.; De Jong, W.; Zijverden, M.V.; Sips, A. y Geertsma, R.E. (2009) “Nanosilver, a review of available data and knowledge gaps in human and environmental risk assessment”. Nanotechnology, 3:109-138.

Yonghong, W.; Xiaoxiao, H. y Keming, W. (2009) “Barbated Skullcup herb extract-mediated byosynthesis of gold nanoparticles and its primary application in electrochemistry". Colloids and Surfaces B: Biointerfaces, 73: 75-79.

Zhang, Y. y Wang, F. (2007) "Use of nanoparticles as building blocks for bioapplications". Molecular Building Blocks for Nanotechnology. Topics in Applied Physics, 109: 353376.

Zhiya-Sheng y Yang-Liu (2011) "Effects of silver nanoparticles on wastewater biofilms". Water Reserch, 45: 6039-6050. 